\title{
Impacts of Application Usage and Local Hardware on the Throughput of Computer Networks with Desktop Virtualization
}

\author{
Vitor Chaves De Oliveira, \\ Alexandre De Assis Mota and Lia Toledo Moreira Mota \\ Department of CEATEC, Electrical Engineering Faculty, \\ Pontifical Catholic University of Campinas-São Paulo, CEP 13086-900, Brazil
}

Received 2012-09-15, Revised 2012-09-15; Accepted 2013-03-13

\begin{abstract}
Currently, virtualization solutions are employed in the vast majority of organizations around the world. The reasons for this are the benefits gained by the approach, focusing on increases in security, availability and data integrity. These privileges are also present in a new technique, which emerges from this same concept and is called desktop virtualization. This method, compelled by these advantages, has grown considerably and is likely to be implemented on more than three-quarters of organizations before 2014. As it is a technique based on physical client server architecture, it conducts all its actions on a local computer and responds to user interaction, through clients that are physically elsewhere. This means that the technique depends on the communication network which makes the interaction possible. Therefore, the importance of the network is increased and it is important to study its behavior compared to a traditional desktop solution, that is, a local solution. This article demonstrates the impact related to a Quality of Service (QoS) parameter, throughput, which suffered great alterations depending on the implemented computational environment. Concomitantly, other results are expressed concerning the Quality of Experience (QoE) decay with a thin client and a significant benefit of virtualization on the QoS, when remote access is required.
\end{abstract}

Keywords: Desktop Virtualization, Quality of Service (QoS), Quality of Experience (QoE), Computer Networks, Thin Client, Client Server Architecture

\section{INTRODUCTION}

During the past decade, virtualization solutions, especially in servers, have grown significantly (Thibodeau, 2012). This is due to several advantages provided by the approach; which according to (Menasce, 2005), are basically increases in security, reliability, availability and scalability, together with a significant cost reduction. Currently, the growth of this method presents a new technique: desktop virtualization, or Virtual Desktop Infrastructure (VDI). Conceptually, this practice is similar to server virtualization and has been praised for bringing similar benefits. However, there is a feature for this use to be implemented, which is relevant: the communication network between the central elements and access clients. In this article, it will be outlined a prognosis of how to assess the viability of this type of implementation, which constitutes the main contribution of this study.

VDI technology is physically based on the use of clientserver architecture; which along with the implementation of desktop virtualization sets personal computers on one or more physical machines. The following sketches (Fig. 1 and 2) illustrate the operation of the technology, physically and logically, showing the various existing layers.

Figure 1 indicates the basic functioning of a clientserver architecture. From it, one can extract that clients are totally dependent of two main things: one is the server that processes their requests and the other is the communication network that physically separates them. Pontifical Catholic University of Campinas-São Paulo, Brazil 


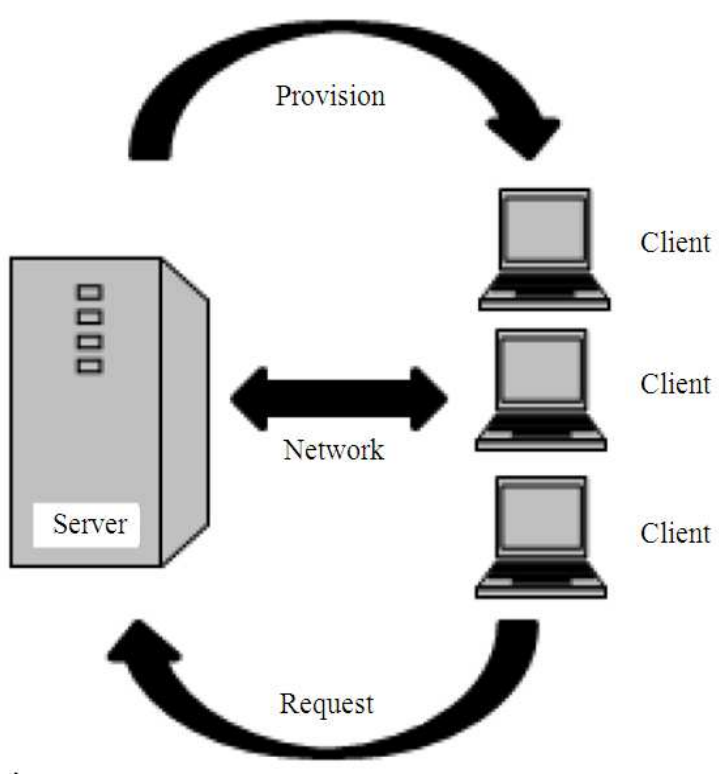

Fig. 1. Client-server architecture

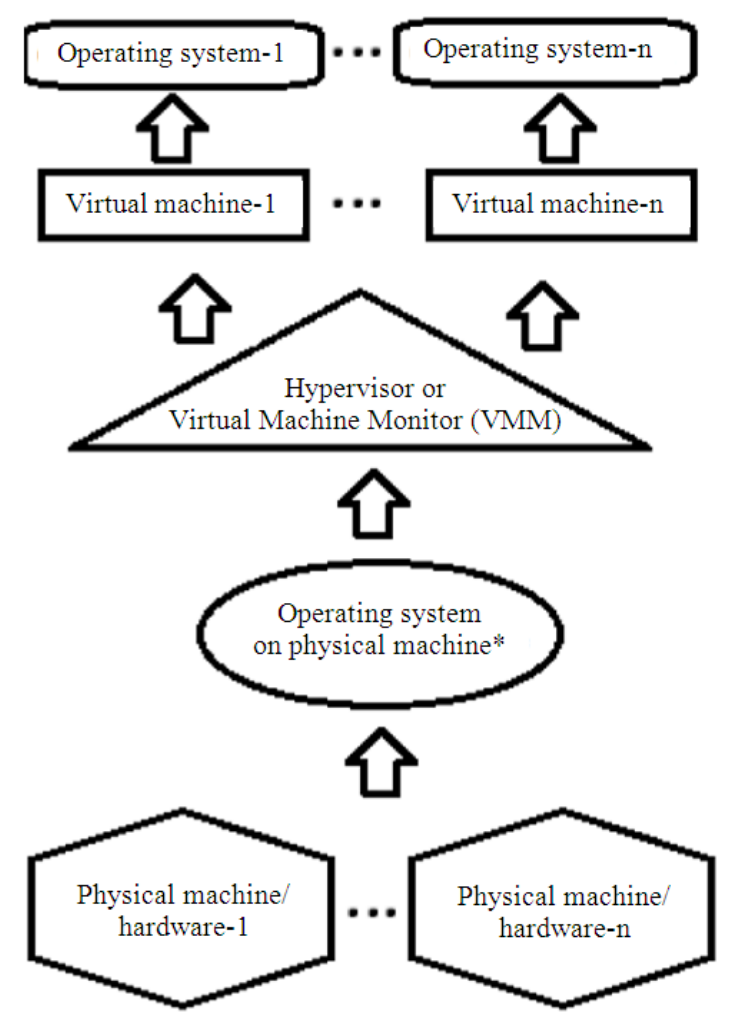

*May or may not be present

Fig. 2. Computational Virtualization representation
This network, when talking about VDI, brings a concern regarding the quality of service of the communication link, once, to have a high quality desktop experience for a user, QoS parameters such as throughput are crucial (Silva, 2004; Adibi et al., 2010; Mota et al., 2011; Biazotto et al., 2011). As for the desktop virtualization, Fig. 2 correctly explains its operation.

At first, there is the hardware, which is the machine itself with all its physical components, such as processors, memory, hard drives, network cards, then, there is a layer of software called hypervisor or Virtual Machine Monitor (VMM), which is the basis of computational virtualization; since, from it, one can create a virtualized hardware. That is, it enables the creation of virtual machines capable of hosting a complete operating system (software), creating the main focus of this article, the Virtual Desktop. It is important to note that may or may not exist an operating system installed directly on the hardware that supports the VMM. That means it's possible to implement an intermediate software layer by installing an OS between the hardware and the hypervisor as illustrated on Fig. 2 (CITRIX, 2011).

The main goal of this study is to analyze the use of VDI by measuring a QoS parameter, throughput and therewith, outline an assessment concerning application usage attendance and local hardware equipment variation, in order to evaluate the feasibility of a VDI implementation.

\section{MATERIALS AND METHODS}

As seen previously, technical standards of QoS are critical for evaluating the possibility of implementing desktop virtualization. Therefore, it was designed a trial which saw throughput (amount of data) of a transmission channel on a VDI environment. For this, it was used the hardware and software components as described below. It's important to point out that the implemented test bench was designed to collect network information at the physical layer. That is, capture all the traffic on Ethernet encapsulation, information acquired directly at the embedded network card on the server.

\subsection{Physical Machine}

Called Server with Operating System (OS) installed directly on the hardware. On top of this OS, it was settled the Hypervisor, through which it was created a virtual machine with another OS installed, creating the Virtualized Desktop. Additionally, at the OS of the physical machine, it was installed a software capable of monitoring the Ethernet network card. Specifications: Server: Dell Vostro 260s Slim ${ }^{\circledR}$, features: Processor: 2nd Generation Intel ${ }^{\circledR}$ Core ${ }^{\text {TM }}$ i5-2400 (3.1 GHz up to 
3.4 GHz with Turbo Boost 2.0, 4 Threads, 6MB Cache, 64 bits) Memory: 6 GB DDR3 SDRAM at $1333 \mathrm{MHz}$, Hard Drive: 500GB, SATA (7200 RPM) Video Card: AMD Radeon HD 6450 1GB DDR3; Integrated Network Card: 10/100/1000 Ethernet LAN on system board, dimensions (width, height and depth): $35.0 \times 10.5 \times 45.0 \mathrm{~cm}$ :

- Operating System installed on the physical machine: Server: Microsoft Windows Server (B) 2008 R2 Enterprise (64bits)

- Software to Monitor Network Interface: Installed on the server: WireShark Network Protocol Analyzer ${ }^{\circledR}$ Version 1.7.0

- Hypervisor Software: Installed on Server: VMware Workstation ${ }^{\circledR} 8$.

- Virtualized Hardware (created from the Hypervisor): Processors: 4, Memory: 4GB, HDD: 60GB, Network Card: Ethernet

- Operating System Installed on the Virtual Machine: Microsoft ${ }^{\circledR}$ Windows 7 Ultimate (64bits)

- Software for Media Playback-installed on all OS of this experiment, except the Thin Client's OS: VLC Media Player 2.0.3

\subsection{Client's Physical Machine-Thin Client Access Terminal}

Model: NC600W, Manufacturer: Net Computer Technology Co. Ltd., Network Card Integrated: 10/100/1000 Ethernet LAN on system board, Memory: 20MB RAM, processor: 533Mhz, native OS: Microsoft Windows ${ }^{\circledR}$ CE 5.0, Dimensions (width, height and depth): $11.9 \times 11.9 \times 2.5 \mathrm{~cm}$.

\subsection{Client's Physical Machine-High Performance Access Terminal (Fat Client)}

Manufacturer: Dell Model: Dell Inspiron N4050 Laptop, Integrated Network Card: 10/100/1000 Ethernet LAN on system board, Memory: 4 GB DDR3 SDRAM at $1333 \mathrm{MHz}$, Processor: 2nd Generation Intel ${ }^{\circledR}$ Core TM i32350M (2.3GHz Threads, 3MB Cache) OS:: Microsoft Windows 7 Home Basic (64bits) ${ }^{\circledR}$; Dimensions (width, height and depth ) $34.0 \times 2.3 \times 24.0 \mathrm{~cm}$.

\subsection{Connection (Transmission Channel)}

Standard CAT5 network cable, with RJ45 connectors.

In order to assess QoS parameters and more specifically, throughput, in an environment that uses virtualized desktops, it is necessary to have a reference regarding the network behavior without using the means of desktop virtualization. Thus three different setups were implemented in order to run the necessary tests to assess the influence of hardware virtualization impacts in the network throughput.
Therefore, in the first setup was implemented an environment without virtualization and with no remote access session, performing a common application for a desktop and accessing server data via Remote File Access. After that, still without the use of virtualization, but using remote access, in a second setup a session of the Server's OS was opened and the application was repeated. Finally, with the results from the previous tests, the application was executed using the virtualized desktop through a remote access session, characterizing a third setup.

The target application in all conducted tests was media playback of a high quality $60 \mathrm{sec}$ MPEG-4 video, for all described setups. All remote access sessions used Remote Desktop Protocol (RDP). The first setup used only the Fat Client because it depends heavily on local hardware structure to run the tests and the Thin Client lacks the required hardware resources. In this context, five tests were carried out in the proposed setups:

\subsection{Test 1}

In the first case, referred to as "Fat_Client_Remote Video_Access" held only one video transmission using the local physical structure. For this, it has connected to the server and the FatClient through the program VLC Media Player, installed on both Operating Systems: Server and Fat Client, which originated video playback. This video file was at the Server's Hard Disk and was accessed by the client, starting the transmission. The moment that playback started, monitoring the server's network interface also started.

\subsection{Test 2}

In the second case, entitled: "Fat_Client_Accessing_Physical_Desktop", the FatClient held a transmission session via Remote Desktop Protocol (RDP) to the Server's OS and played the same video.

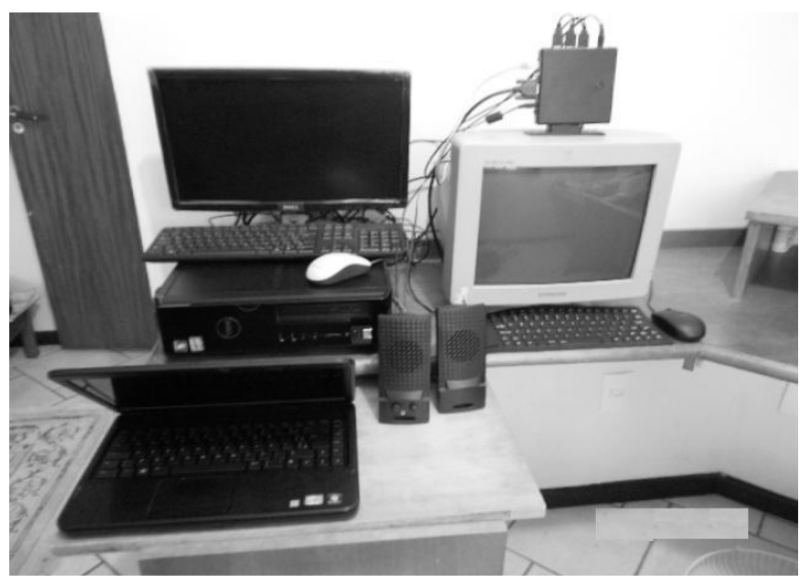

Fig. 3. Experiment setup (bench picture) 
In this experiment, the video itself was not physically transmitted from one machine to another as in the previous test. It was played on one machine (Server) and displayed in another (FatClient).

\subsection{Test 3}

The same implementation as the second test occurred on the third experiment called: "Thin_Client_Accessing_Physical_Desktop" with a difference: the access client is modified to the ThinClient.

\subsection{Test 4}

The forth

experiment,

named: "Fat_Client_Accessing_Virtual_Desktop" occurred through the prism of desktop virtualization technology. This environment was implemented as follows: on the Server's OS-it was installed a program that allows the creation of virtualized hardware, the hypervisor, termed as VMWare WorkStation. With this software, a virtual machine was made, whose resources have already been detailed above. On this virtual machine, it was installed an OS and a Media Playback Software (VLC). It's important to note that it was also placed at Virtual Desktop's Hard Drive the mentioned video file to be displayed. The Virtual Desktop is accessed by both clients through a session via RDP protocol and then the video is played.

\subsection{Test 5}

The same implementation as the forth test occurred on the fifth experiment called: "Thin_Client_Accessing_Virtual_Desktop" with a difference: the access client is modified to the Thin Client.

Moreover, it is noteworthy that the Software WireShark was configured to monitor the same physical interface for all measurements. In addition, it must be distinguished that all tests occurred separately and on the tests without virtualization, the virtual machine, along with all its components was completely deactivated. Figure 3 shows the setup experiment, it's a picture of the bench.

\section{RESULTS}

Table 1 and Fig. 4 elucidate the results obtained for the various experiments. An important point observed during the experiments was that for all environments ran with the Thin Client, the application performance was totally unsatisfactory, both for the image and for the audio. And for all tests conducted with the Fat Client, the opposite occurred, because on these tests the behavior of the application was excellent, the environment, virtualized or not, was "transparent" to the user's system, i.e., imperceptible. With this result and this observation one can draw some conclusions about local hardware required for a VDI and on the behavior of the communication network, which will be presented as follows.

\section{DISCUSSION}

Following are the analysis of the results related to three different comparisons: Local Desktop versus Virtualized Desktop, Robust client's hardware (Fat Client) versus Reduced client's hardware (Thin Client) and Remote Access of a Physical Machine versus Remote Access of a Virtual Machine. Exploring the results, it is observed that the average throughput of a conventional solution (local) desktop is increased in $18,5 \%$ once a virtual desktop structure is employed. Figure 5 illustrates this situation.

It is necessary to inform that a local solution allows a simplified data transmission operation, which demands less QoS in a network, since this solution allows the use of a buffer, which storages packets received at different times in memory in order to maintain synchronization for later reading. Whereas in a virtualized desktop environment, there is no way to use a buffer, because the system has to be real time, responding to user interaction. In other words, the reason is that it isn't possible to know what data the user will access next, for example: if the user will continue watching a video or reading a document. Another important point is the fact that desktop virtualization demands local hardware sufficient to treat the frames in real time. This means that it's necessary to look at this matter relating its dependence with the applications that the user will need. Therefore, it is concluded that simple and inexpensive access terminals, such as Thin Clients may not be a viable option when it comes to VDI, including further network load increases. Figure 6 illustrates these results. It can be observed that the reduced client resulted in an average throughput of about $130,0 \%$ higher than the robust client. Meanwhile, it's essential to recall that the Thin Client, despite of the greater network consumption, the Quality of Experience was very poor.

Another interesting characteristic detected by these experiments was the benefit in having virtualized hardware if remote access is mandatory. Figure 7 accessing a virtual desktop rather than a physical desktop. Thus, is acknowledged that it is advantageous to virtualized when remote access is a must, such as remote access to servers in an organization. 
Vitor Chaves De Oliveira et al. / American Journal of Applied Sciences, 10 (1): 117-122, 2013

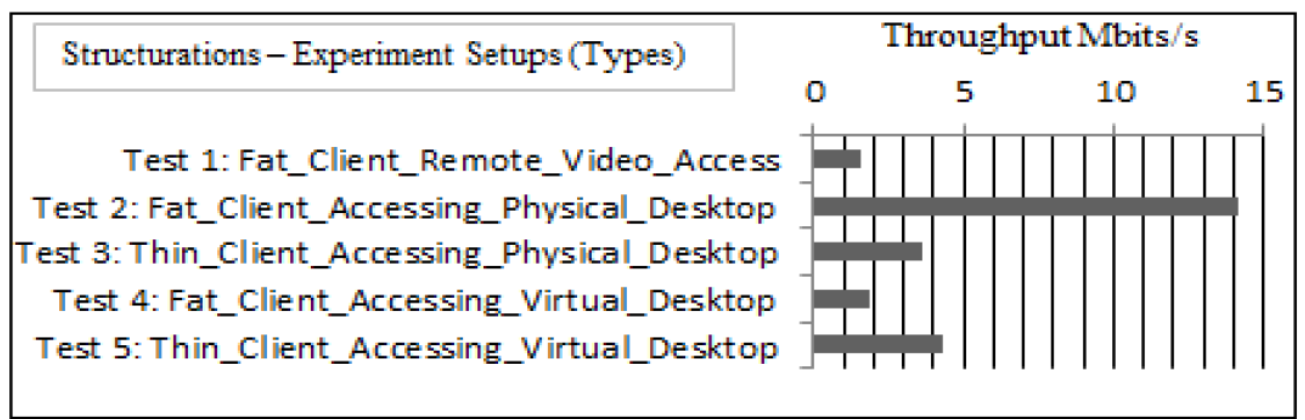

Fig. 4. Average throughput in Mbits per second at the Server's physical network adapter

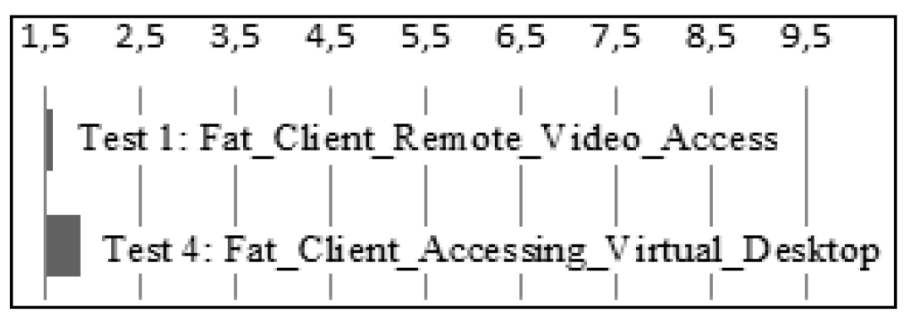

Fig. 5. Physical desktop structure versus virtual desktop structure

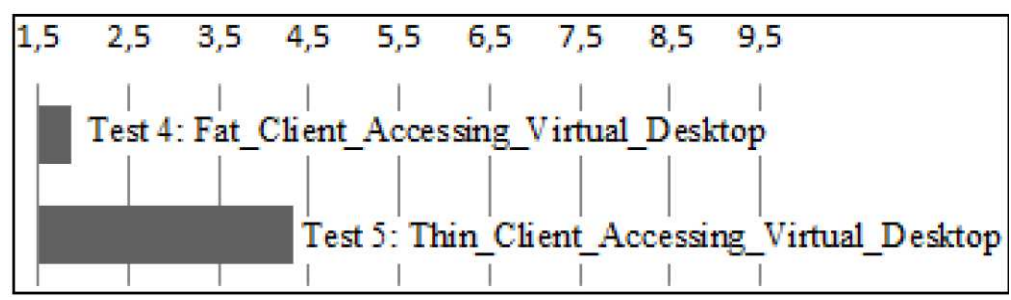

Fig. 6. Robust client hardware versus Reduced client hardware on a VDI

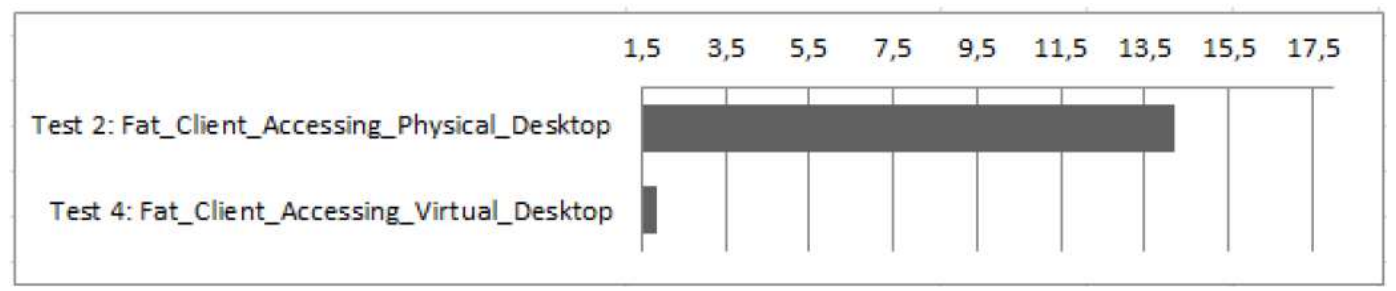

Fig. 7. Remote access to physical machine versus remote access to virtual machine

Table 1. Average throughput in Mbits per second at the Server's physical network adapter

Structurations-experiment setups (Types)

Test 1: Fat_Client_Remote_Video_Access

Test 2: Fat_Client_Accessing_Physical_Desktop

Test 3: Thin_Client_Accessing_Physical_Desktop

Test 4: Fat_Client_Accessing_Virtual_Desktop

Test 5: Thin_Client_Accessing_Virtual_Desktop
Throughput Mbits/sec

3,600

1,883

4,326 
This is justified once the throughput values, in Mbits/sec, of Test 2: Fat_Client_Accessing_Physical_Desktop and Test 4: Fat_Client_Accessing_Virtual_Desktop are compared. The data on Fig. 7 shows that the approach with desktop virtualization presents a throughput value of $1,883 \mathrm{Mbits} / \mathrm{s}$, that is about $87 \%$ less than the obtained without virtualization technology, which was $14,195 \mathrm{Mbits} / \mathrm{s}$.

\section{CONCLUSION}

From the results obtained, it is concluded that desktop virtualization brings benefits tangents; mainly to security, availability and integrity of information. However, one should assess whether these benefits outweigh the additional cost (overhead) imposed on the communication network, as this approach will require constant monitoring and real-time QoS parameters, which will certainly be supported by a supervisory system, that will control and monitor the network with a high level of automation. Furthermore, it is seen that the function of specialized support personnel on computer networks have increased their importance to this approach. The reason is simple: to ensure that the quality of the user experience for the VDI is transparent and one way to assign a deployment consisting of an exponentiated attention to the communication channel.

In this article, it has been pointed out the high cost of desktop virtualization in communication network and it encourages you to think differently about VDI, concerning levels of segregation. In light of these facts, this study proposes the analysis of virtualization classified as hybrids, which may prove more interesting. That is, it would be more convenient to devise a mixed design and/or cross-virtualization local operations (clients). Therefore, it is deemed essential to analyze that probably one should choose which applications must be performed locally and which would need to be virtualized. Thus, possibly, would facilitate the satisfactory fulfillment of requirements for security, availability and data integrity and the cost of virtualization on the network would be reduced. Following this line of reasoning, one might think, including the variation of the local hardware, balancing it with the application that you use. In addition, other QoS parameters and energy consumption and network communication should be evaluated so that a more accurate diagnosis regarding the implementation of desktop virtualization can be outlined.

\section{REFERENCES}

Adibi, S., R. Jain, S. Parekh and M. Tofighbakhsh, 2010. Quality of Service Architectures for Wireless Networks: Performance Metrics and Management. 1st Edn., Information Science Reference, Hershey, PA, ISBN-10: 9781615206803, pp: 691.

Biazotto, L.H., A.J.F. Peris, F.S. Cyriaco, O.C. Branquinho and A.A. Mota et al., 2011. A mapping energy consumption method in telecommunications networks. Proceedings of the XL IGIP-International Symposium of Engineering Education, (PISEE' 11), pp: 700-703.

CITRIX, 2011. Desktop virtualization and security: A global market research report. Citrix Systems Inc.

Menasce, D.A., 2005. Virtualization: Concepts, applications and performance modeling. Proceedings of the International CMG Conference, (CC' 05), pp: 407-414.

Mota, L.T.M., A.D.A. Mota and L.F. Fontolan, 2011. Quality of service policy for IEEE 802.11 networks with service rate selection based on fairness index. J. Comput. Sci., 7: 600-604. DOI: 10.3844/jcssp.2011.600.604

Silva, D.J., 2004. QoS Assessment in Corporative Networks (in portuguese). MSc Dissertation.

Thibodeau, P., 2012. Server virtualization pushes storage demand to new highs. ComputerWorld. 